\title{
Determination of Cardiac Ejection Fraction by Electrical Impedance Tomography using Simulation Models and Computational Intelligence
}

\author{
Tales Fonseca ${ }^{a 1}$, Luciana Campos $^{a}$, Franciane Peters ${ }^{b}$, Luis Paulo da Silva \\ Barra $^{a}$, Rodrigo Weber dos Santos ${ }^{a}$ and Leonardo Goliatt ${ }^{a}$ \\ ${ }^{a}$ Federal University of Juiz de Fora, Juiz de Fora, Brazil \\ ${ }^{b} \mathrm{COPPE}$ - Federal University of Rio de Janeiro, Rio de Janeiro, Brazil \\ Received on December 01, 2016 / accepted on May 12, 2017
}

\begin{abstract}
The main function of the heart is to pump blood to all areas of the body and the proper occurrence of this process is essential for health. The ejection fraction is an important clinical parameter to determine the amount of blood pumped by the heart in each cardiac cycle. A value outside the normal range indicates that the heart is contracting abnormally. Combining low cost and high portability, Electrical Impedance Tomography with Regression Models is an alternative to obtain continuous estimations of cardiac ejection fraction, allowing a quick diagnosis on the health of the heart. This paper presents four computational intelligence models to estimate the ejection fraction using the electrical measures from Electrical Impedance Tomography: Multilayer Perceptron, Extreme Learning Machine, Random Forests and Elastic Net Regressor. A simulated dataset is used to train and assess the performance of all models. In order to improve the model's performance, an exhaustive search procedure on the parameters of each model is implemented. The overall evaluation of the results show that all models achieved percentage errors below $2 \%$ and the neural networks have produced better averaged predictions.
\end{abstract}

Keywords: Ejection Fraction, Computational Intelligence and Simulation Models

\section{Introduction}

For analyzing the efficiency of the heart is used an important clinical parameter called cardiac ejection fraction $(\mathrm{EF})$. This parameter represents

\footnotetext{
${ }^{1}$ E-mail Corresponding Author: tales.lima@ice.ufjf.br
} 
the amount of blood pumped out of each ventricle in each cardiac cycle. Clinically, it is more common to consider only the ejection fraction of the left ventricle. By definition, the EF is calculated as follows:

$$
E F=\frac{P V}{E D V}=\frac{E D V-E S V}{E D V}
$$

where PV represents the volume of blood pumped, obtained by the difference between the end-diastolic volume (EDV) and the end-systolic volume (ESV).

To simplify the calculation of EF, instead of using the volume of the ventricle in diastole and systole cycles, the areas can be used without loss of quality. The areas that represent the ventricles are based on a cross section of the heart cavities and it is assumed to be proportional to their volumes. Thus, the EF can be calculated as follows:

$$
E F=\frac{E D A-E S A}{E D A}
$$

where EDA is the area of the end of diastole, while ESA means the area of the end of systole.

The cardiac ejection fraction is considered an important clinical parameter because its high correlation with the functional state of the heart. The value of change in left ventricular ejection fraction change are associated to some heart diseases, such as aortic regurgitation [1-3] and identifies highrisk patients, who are likely to benefit from a more aggressive therapeutic strategy [4]. There are several non-invasive techniques that can be applied to determine the EF as echocardiogram, cardiac catheterization, cardiac magnetic resonance, computerized tomography, and others. Although such techniques are capable of producing high definition images for well-accurate diagnostics, they can not be used for continuous monitoring, especially due to their high costs. In this work, four methods for continuous monitoring of cardiac ejection by Electrical Impedance Tomography (EIT) is used. The reason of it is that the EIT technique does not use ionizing radiation, has low costs and better portability.

The Electrical Impedance Tomography produces an image of the conductivity distribution of a body part, using measures of current injection and potential protocols taken on the boundary of the domain. Typically, conducting electrodes are attached to the patient's body and small currents are applied. Mathematically, the EIT is classified as a non-linear, ill-posed and ill-conditioned inverse problem. In EIT inverse problem one wants to find the electrical resistivity of the body using measures of electrical potential on the boundary excited by known electrical current [5]. Several aspects 
involved in the solution of the EIT problem were discussed in [5-7]. In the context related to biomedical engineering, recent work [7] has discussed the feasibility of Electrical Impedance Tomography for continuous monitoring of cardiac ejection fraction, and other related works [6] have shown preliminary results on the same subject.

In this paper we propose the use of computational intelligence models to replace Finite Element Analysis [8] or Boundary Element Analysis [7] in the determination of Ejection Fraction. We employed a Multilayer Perceptron Neural Network, an Extreme Learning Machine, Random Forests and Elastic Network Regression. We compare their performance with the computational intelligence methodologies $[9,10]$ and simulation strategies [7] that deal with the same problem. All the tests were carried out based on a synthetic dataset generated on Reference [7]. The results found in this paper are competitive with those found on the literature and suggest that Multilayer Perceptron Neural Network and Extreme Learning Machine promise alternatives as diagnostic tools that offer continuous and non-invasive estimations of cardiac ejection fraction.

\section{Materials and Methods}

\subsection{Simulated Dataset}

Because of the lack of a real medical database, we have used a synthetically generated dataset from a parametrization from an image of a human torso model provided by magnetic resonance. This dataset contains 209 samples simulating various types of synthetic heart configuration (with anomalies or not). Each sample has 104 measures of the electrical potentials associated with two areas of the left ventricle, referring to End of Diastole and Systole. Both areas are necessary to the EF's calculation. Details of the generated dataset can be found in [7].

\subsection{Regression Models}

Four regression models were used to approximate the End of Diastole Area $(E D A)$ and the End of Systole Area $(E S A)$ from simulated dataset: Extreme Learning Machine Regressor, Elastic Net Regression, Random Forests and Multilayer Perceptron Neural Networks. Detailed description of the methods employed here and their parameters can be found in [11] and $[12]$. 


\subsection{Parameter settings using Cross-validation}

Cross-validation is a sampling statistical technique to evaluate the ability of generalization of a model from a dataset [13]. Among the cross-validation techniques, one of the most used is the called K-Fold[14]. K-Fold uses a part of the data available to fit the model, and another different part to test it. A dataset containing $N$ samples are randomly divided into $\mathrm{K}$ subsets, where $\mathrm{K}>1$. From the $\mathrm{K}$ subsets, $\mathrm{K}-1$ are used for training and the remaining set is used for testing. This process is repeated $\mathrm{K}$ times, using a different test set in each iteration. Figure 1 shows an example of the dataset divided into $\mathrm{K}=7$ subsets in each iteration of the cross validation process.

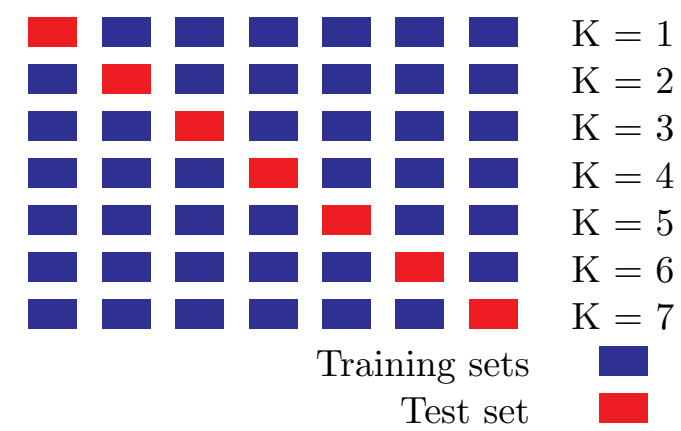

Figure 1: Division of the dataset in $\mathrm{K}=7$ subsets (folds).

The parameters that are not learned directly in the model training process, called hyperparameters, were set by an exhaustive search (grid search) [11] on the hyperparameter space to find the set of parameters that leads to the best performance of cross validation. Table 1 shows the set the hyperparameters used in this paper for each model.

\subsection{Performance Metrics}

In order to assess the predictive performance of the models, we have used the following evaluation metrics: $R^{2}$ score, Mean Squared Error $(M S E)$, Model Efficiency $(M E F)$ and Mean Absolute Percentage Error (MAPE). These criteria are given according to

$$
M S E=\frac{1}{N} \sum_{i=0}^{N-1}\left(y_{i}-\hat{y}_{i}\right)^{2}, \quad R^{2}=1-\frac{\sum_{i=0}^{N-1}\left(y_{i}-\hat{y}_{i}\right)^{2}}{\sum_{i=0}^{N-1}\left(y_{i}-\bar{y}\right)^{2}}
$$


Table 1: Model parameters used in grid search with cross validation.

\begin{tabular}{lll}
\hline Model & Parameters (see Ref. [11, 12]) & Parameters Range \\
\hline ELM & Activation function & $\begin{array}{l}\text { Sigmoid, Hyperbolic Tangent, } \\
\text { Gaussian, Multiquadric, }\end{array}$ \\
& & $\begin{array}{l}\text { Inverse Multiquadrics } \\
\end{array}$ \\
& Neurons in the hidden layer $(L)$ & $5,10,20,30,50,100,150,200$ \\
\hline ENR & alpha $(\alpha)$ & $1,10^{-1}, 10^{-2}, 10^{-3}, 10^{-4}$, \\
& & $10^{-5}, 10^{-6}$ \\
& $0.70,0.80,0.85,0.90,0.95$, \\
& $L_{1}$-ratio $(\rho)$ & $0.98,1.00$ \\
\hline RF & Ratio between the number & $1.0,0.3,0.1$ \\
& of selected variables and the & \\
& number of variables $\left(r_{\text {try }}\right)$ & \\
& Minimum samples leafs $\left(n_{\text {leaf }}\right)$ & $1,3,5,9,17$ \\
& Number of trees $\left(n_{t} r e e\right)$ & 200 \\
\hline MLP & Topology & $(1)$ single feedforward and $(2)$ \\
& & fully conected feedforward \\
& Hidden layers & {$[2],[3],[4],[5],[10],[2,2],[3,2]$,} \\
& & {$[5,2],[5,5]$} \\
\hline
\end{tabular}

$$
M E F=1-\frac{(N-1) \sum_{i=0}^{N-1}\left(y_{i}-\hat{y}_{i}\right)^{2}}{(N-p) \sum_{i=0}^{N-1}\left(y_{i}-\bar{y}\right)^{2}}, \quad M A P E=\frac{1}{N} \sum_{i=0}^{N-1} \frac{\left|y_{i}-\hat{y}_{i}\right|}{\left|y_{i}\right|}
$$

where $N$ is the number of samples, $p$ is the number of model parameters, $\hat{y}_{i}$ is the estimated target output, $y_{i}$ is the corresponding (correct) target output and $\bar{y}$ is the mean of the vector $\left[y_{1}, \ldots, y_{N}\right]$.

\section{$3 \quad$ Results and Discussion}

In this section we present and discuss the results obtained for the regression models described in Section 2. Regression models return areas as output, and then they are used to calculating the EF. In all computational experiments we have used 11-fold cross-validation, and in order to obtain consistent and reliable metrics a total of 30 runs were executed with different random seeds in each one. In each run, the dataset is shuffled to assure different folds in cross validation. In order to obtain reliable performance metrics all results are averaged (over 30 runs) and standard deviations are 
shown. The following results were obtained with implementations based in packages provided by [11] and [12].

The percentage error of the End Diastolic Area $(E D A)$ and the End Systolic Area $(E S A)$ of all models are showed in Figure 2. As it can be observed in the boxplots depicted in this figure, among all prediction models, RF obtained the worst performance. For EDA, MLP has achieved a slightly better performance when compared to ELM. Both regression methods MLP and ELM have obtained similar mean relative percentage errors for $E S A$, as can be seen in Figure 2 on the right, however ELM has produced results with smaller interquartile range.

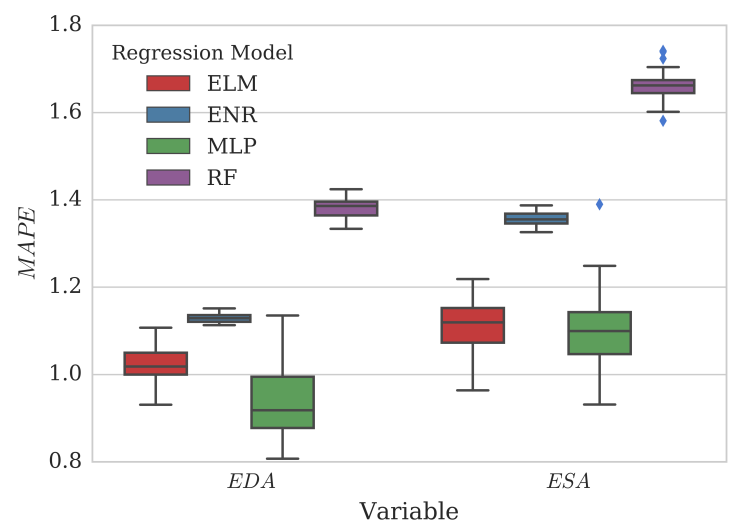

Figure 2: Relative errors for End of Diastole Area (left) and End of Systole Area (right). The blue dots are outliers.

Table 2 summarizes the performance metrics for all models. The first column shows the regression models, the second column shows the Mean Efficiency $(M S E)$ while the Mean Squared Error $(M S E)$ and $R^{2}$ score appears in the third and last columns respectively. Satisfactory agreement among the model predictions and simulates data was obtained from the analysis. The comparisons between MLP, ELM, RF and ENR indicate that the best results in terms of the $R^{2}$ values were obtained from the MLP and ELM. Averaged $R^{2}$ values of the relationship between simulated data and predicted data are 0.9552 and 0.9528 , respectively. When the models are compared in terms of the $M S E$ (a measurement of variance around the regression line), it can be seen that the lowest averaged $M S E$ is obtained from MLP followed by ELM. The other models RF and ENR give higher $M S E$ values. A similar behavior can be observed for averaged values for Model Efficiency $(M E F)$ : MLP obtained an averaged $M E F$ equals to 
0.9958 and ELM produced an averaged value of 0.9960 , while RF and ENR presented lower $M S E$ values.

Table 2: Comparison of the performance metrics for all models. Each column shows the averaged results and standard deviations inside the parenthesis. For each regression model, a total of 30 runs were executed with different random seeds in each one.

\begin{tabular}{clll} 
Model & $R^{2}$ & $M S E$ & $M E F$ \\
\hline ELM & $0.9528(0.004572)$ & $0.0003(2.904 \mathrm{e}-05)$ & $0.9960(0.0002871)$ \\
ENR & $0.9335(0.001214)$ & $0.0004226(7.714 \mathrm{e}-06)$ & $0.9944(6.166 \mathrm{e}-05)$ \\
MLP & $0.9552(0.007116)$ & $0.0002844(4.52 \mathrm{e}-05)$ & $0.9958(0.001313)$ \\
RF & $0.8897(0.003902)$ & $0.0007008(2.479 \mathrm{e}-05)$ & $0.9904(0.00024)$ \\
\hline
\end{tabular}

The comparison of $M S E$ and $R^{2}$ performance metrics for Ejection Fraction calculated using Eq. (2) is presented in Figure 3. Comparing the Figures 2 and 3 one can observe that the models with the lowest percentage errors regarding the variables $E D A$ and $E S A$ were also the same models that obtained the best performance in the $M S E$ metric to calculate the EF.
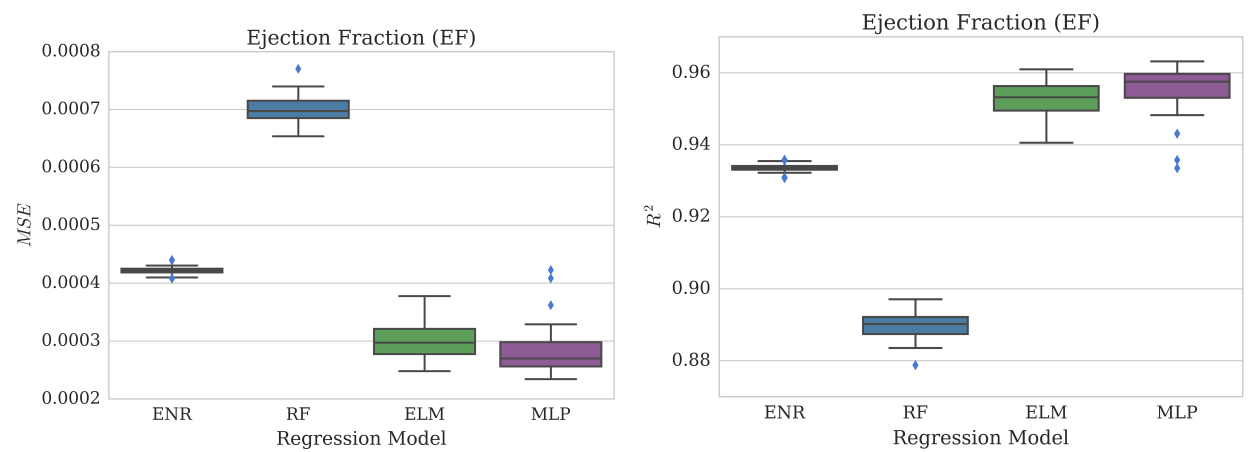

Figure 3: Comparion of the performance of regression models according to $M S E$ (left) and $R^{2}$ (right).

Table 3 shows the parameters of the best regression models according to $M S E$. While Table 2 shows the averaged performance for 30 runs, table 3 presents the set of parameters for each model that produced the lowest value of $M S E$ in all runs.

In order to perform a comparative study between the regression models presented here in this paper and the techniques used in [5] and [9], in Table 
Table 3: Parameters of the regression models with the best performance according to $M S E$ performance metric.

\begin{tabular}{ccl}
\hline Model & $M S E$ & Parameters \\
\hline ELM & 0.00026475 & $L=100$, activation function $=$ Multiquadrics \\
ENR & 0.00041357 & $\alpha=1 \mathrm{e}-06, L_{1}$ ratio $=1.0$ \\
MLP & 0.00023405 & connectivity $=$ feed-forward, hidden layer $=[5,5]$ \\
RF & 0.00067224 & $n_{\text {try }}=0.1, n_{\text {leaf }}=1$ \\
\hline
\end{tabular}

4 we show the comparison of the percentage error for $E D A$ and $E S A$. The values obtained by regression models for $E D A$ were considerably better than the values of the reference. The comparisons between the regression models indicate that the best results were obtained from MLP and ELM models, as well as the results already presented. In relation to $E S A$ the references [5] and [9] obtained better results, especially the reference [5], but in comparison between the regression models, again the MLP and ELM models obtained better results. It is important to note that the results presented by [5] was obtained through simulations and the result of this work is averaged in 30 runs. The result of the best run of MLP showed a percentage error equal to 1.02 to $E S A$, which is a better result than in [9].

Table 4: Comparison of the percentage error for EDA and ESA. Each column shows the averaged results and standard deviations inside the parenthesis. For each regression model, a total of 30 runs were executed with different random seeds in each one.

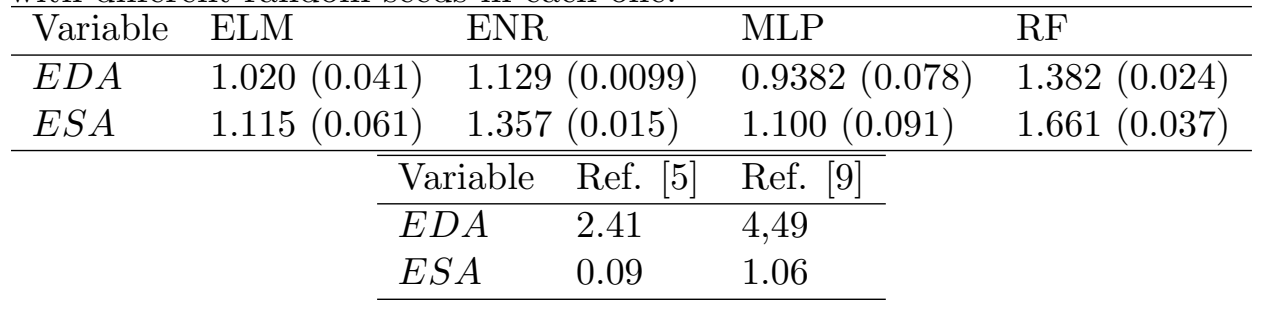

\section{Conclusions}

The results obtained from all models developed herein are in satisfactory agreement with the simulated results in the database. The comparison between regression models described in Section 2 indicated that the MLP and ELM models markedly outperform the RF and ENR models. This was 
evidenced by performance metrics used for evaluating the models. The MLP and ELM models produced higher coefficient of determination $R^{2}$ and $M E F$ values, and lower $M S E$ values.

This paper introduces a grid search with cross validation strategy on the hyperparameters of the four regression methods, and evaluate MLP with a single feedforward networks and fully connected networks. The overall evaluation of the results obtained throughout the study revealed that the MLP and ELM perform the better average prediction ability than RF and ENR models. We also conclude that ELM (Extreme Learning Machines) produced competitive results when compared to MLP networks for all metrics. However, the learning procedure of ELM is cheaper than MLP training involving a simple low-dimension linear regression instead of solving a high-dimensional optimization problem as it happen in MLP networks.

\section{References}

[1] R. Bekeredjian and P. A. Grayburn. Valvular heart disease: aortic regurgitation. Circulation, 112(9):125 - 134, 2005.

[2] Bharathi Upadhya, George E. Taffet, Che Ping Cheng, and Dalane W. Kitzman. Heart failure with preserved ejection fraction in the elderly: scope of the problem. Journal of Molecular and Cellular Cardiology, 83:73 - 87, 2015. Perspectives on Cardiovascular Aging: Top to Bottom.

[3] Madelien V. Regeer, Michel I.M. Versteegh, Robert J.M. Klautz, Martin J. Schalij, Jeroen J. Bax, Nina Ajmone Marsan, and Victoria Delgado. Comparison of left ventricular volume and ejection fraction and frequency and extent of aortic regurgitation after operative repair of type a aortic dissection among three different surgical techniques. The American Journal of Cardiology, 117(7):1167 - 1172, 2016.

[4] Angelo Squeri, Nicola Gaibazzi, Claudio Reverberi, Maria Michela Caracciolo, Diego Ardissino, and Tiziano Gherli. Ejection fraction change and coronary artery disease severity: A vasodilator contrast stress-echocardiography study. Journal of the American Society of Echocardiography, 25(4):454 - 459, 2012.

[5] Franciane C Peters, Luis Paulo da S Barra, and Rodrigo W dos Santos. Determination of cardiac ejection fraction by electrical impedance tomography. INTECH Open Access Publisher, 2011. 
[6] Luis Paulo da Silva Barra, Franciane C Peters, Cátia de Paula Martins, and Helio José Corrêa Barbosa. Computational experiments in electrical impedance tomography. XXVII Iberian Latin American Congress on Computational Methods in Engineering, 2006.

[7] Franciane C Peters, Luis Paulo S Barra, and Rodrigo Weber dos Santos. Determination of cardiac ejection fraction by electrical impedance tomography-numerical experiments and viability analysis. In Computational Science-ICCS 2009, pages 819-828. Springer, 2009.

[8] BM Rocha, EM Toledo, LPS Barra, and RW Santos. An electromechanical left ventricular wedge model to study the effects of deformation on repolarization during heart failure. BioMed Research International, 2015:1-12, 2015.

[9] RGN Santos Filho, LCD Campos, RW dos Santos, and LPS Barra. $A M I C A I$ 2013, chapter Determination of Cardiac Ejection Fraction by Electrical Impedance Tomography Using an Artificial Neural Network, pages 130-138. Springer Berlin Heidelberg, Berlin, Heidelberg, 2013.

[10] RGN Santos Filho, LCD Campos, RW dos Santos, and LPS Barra. Artificial neural networks ensemble applied to the electrical impedance tomography problem to determine the cardiac ejection fraction. In IBERAMIA 2014, pages 734-741. Springer, 2014.

[11] F Pedregosa, G Varoquaux, A Gramfort, V Michel, B Thirion, O Grisel, M Blondel, P Prettenhofer, R Weiss, and V Dubourg. Scikit-learn: Machine learning in python. The Journal of Machine Learning Research, 12:2825-2830, 2011.

[12] M Wojciechowski. Solving differential equations by means of feedforward artificial neural networks. In Proceedings of the 11th International Conference on Artificial Intelligence and Soft Computing - Volume Part I, ICAISC'12, pages 187-195, Berlin, Heidelberg, 2012. Springer-Verlag.

[13] Ron Kohavi et al. A study of cross-validation and bootstrap for accuracy estimation and model selection. In Ijcai, volume 14, pages 1137-1145, 1995.

[14] Trevor Hastie, Robert Tibshirani, and Jerome Friedman. The Elements of Statistical Learning - Data Mining, Inference, and Prediction. Springer, Verlag, New York, 2 edition, 2009. 